\title{
GROUP THEORETIC PROPERTIES INHERITED BY LOWER CENTRAL FACTORS
}

\author{
by HOWARD SMITH
}

(Received 17 September, 1985)

Several properties are known to be inherited from the derived factor group of a group $G$ by other factors $\Gamma_{i}=\gamma_{i}(G) / \gamma_{i+1}(G)$ of the lower central series. Derek Robinson proved in [1] that the defining property of any class $\mathfrak{X}$ of groups which is closed under the forming of homomorphic images of tensor products is so inherited. Possibilities for $\mathfrak{X}$ here include the following classes.

(1) Finite groups, groups which satisfy the maximal (resp. minimal) condition, minimax groups, min-by-max groups, groups with finite rank, groups with finite $p$-ranks for all $p=0$ or a prime.

The property of being a periodic $\pi$-group (resp. a group with finite exponent dividing some fixed integer $e$ ) is easily seen to be inherited by $\Gamma_{i+1}$ from $\Gamma_{i}$ for any integer $i \geqslant 1$. In Lemma 2.2 of [3] J. P. Williams showed that the property of having finite rank passes from $\Gamma_{2}$ to $\Gamma_{i}(i>2)$, while the lemma in [2] states that if $\Gamma_{n}$ is periodic with finite $p$-rank for all $p$ then so is $\Gamma_{m}$ for all $m>n$. Here we shall prove the following (where $r_{p}(X)$ denotes the $p$-rank and $r_{0}(X)$ the torsion-free rank of an abelian group $X$ ).

THEOREM. (a) If $r_{0}\left(\Gamma_{n}\right)=r<\infty$, then $r_{0}\left(\Gamma_{n+1}\right) \leqslant r^{2} n$.

(b) If $r_{p}\left(\Gamma_{n}\right)=s<\infty$ and $r_{0}\left(\Gamma_{n}\right)=r<\infty$, then $r_{p}\left(\Gamma_{n+1}\right) \leqslant(r+s)^{2} n$.

COROLlary. If $G$ is a group such that $\Gamma_{n}$ belongs to one of the classes $\mathfrak{X}$ listed in (1) above then $\Gamma_{m}$ belongs to $\mathfrak{X}$ for all $m \geqslant n$.

The classes (1) indicate some of the finiteness conditions most commonly referred to. A general result corresponding to that of Robinson on tensor products would be more satisfactory, but $\Gamma_{2}$ may be cyclic while $\Gamma_{3}$ is non-cyclic and so there is no obvious generalisation here.

A rather trivial example points to further obstacles to extending the above theorem.

EXAMPLE. Let $H$ be the group defined by taking the free nil-3 group on generators $a$ and $b$ and adding the relations $a^{25}=1=b^{25}$. For $i=1,2,3$, let $\Gamma_{i}$ denote $\gamma_{i}(H) / \gamma_{i+1}(H)$ and let $x$ be the automorphism of $H$ which maps $a$ to $a^{2}$ and $b$ to $b^{3}$. Then $[a, b]^{25}=1$, $[a, b, a]^{25}=1=[a, b, b]^{25}$ and $\Gamma_{2}$ is cyclic of order 25 and $\Gamma_{3}$ is non-cyclic of order 625 . It is routine to check that $[a, b, x, x]=[a, b, b]^{7}[a, b, a]^{-2}$ and hence that $\left[\Gamma_{2},\langle x\rangle,\langle x\rangle\right]=$ 1. But $[a, b, a, x]=[a, b, a]^{11}$ and $[a, b, b, x]=[a, b, b]^{17}$ generate $\gamma_{3}(H)$ and so $\left[\Gamma_{3},\langle x\rangle\right]=\Gamma_{3}$. Thus $\Gamma_{2}$ is a "polytrivial" $\langle x\rangle$-module but $\Gamma_{3}$ is not.

Proof of the theorem. Suppose $G$ satisfies the hypothesis of part (a) and assume $\gamma_{n+2}(G)=1$. As in [2] we define certain commutators as follows. Let $\sigma=\left[x_{1}, \ldots, x_{n}\right]$ for

Glasgow Math. J. 29 (1987) 89-91. 
some $x_{i} \in G$ and write $\sigma_{1}=x_{1}, \sigma_{i}=\left[x_{1}, \ldots, x_{i}\right]$ for $i=1,2, \ldots, n$. Let $g \in G$ and set $\alpha_{j}=\left[g, x_{n}, \ldots, x_{j}\right], j=1, \ldots, n$, and $\alpha_{n+1}=g$. We can use the Jacobi identity to deduce that

$$
[\sigma, g] \in\left\langle\alpha_{1},\left[\sigma_{n-j}, \alpha_{n-j+2}, x_{n-j+1}\right], \quad j=1, \ldots, n-1\right\rangle .
$$

Now suppose that $\sigma(1), \ldots, \sigma(r)$ are left-normed commutators of weight $n$ which generate a free abelian subgroup of rank $r$ of $\gamma_{n}$ modulo $\gamma_{n+1}$. Writing $S=$ $\langle\sigma(1), \ldots, \sigma(r)\rangle$, we have that $[S, G]$ is generated by elements $[\sigma(k), g]$, and each of these lies in an $n$-generated subgroup $U_{k}(g)$ of the type described in (2). Since $\gamma_{n} / S \gamma_{n+1}$ is periodic, each of the elements $\alpha_{2},\left[\sigma_{n-j}, \alpha_{n-j+2}\right], j=1, \ldots, n-1$ (for each $k$, with the obvious notation) has some power in $S \gamma_{n+1}$ and so there are integers $v_{k}(g)$ such that

$$
\left(U_{k}(g)\right)^{v_{k}(g)} \leqslant\left\langle\left[S, x_{k 1}\right], \ldots,\left[S, x_{k n}\right]\right\rangle=T_{k} \text {, say, }
$$

where $x_{k 1}, \ldots, x_{k n}$ are the entries of the commutator $\sigma(k)$.

Let $T=T_{1} \ldots T_{r}$. Then $[S, G] / T$ is periodic and $T$ is at most $r^{2} n$-generated. It follows that $\gamma_{n+1}$ has torsion-free rank at most $r^{2} n$, thus proving (a).

Now let $T^{*}$ be a free abelian subgroup of maximal rank in $T$. Then the image of $\gamma_{n+1}$ in $G / T^{*}$ is periodic, and in order to prove part (b) we may assume that $\gamma_{n+1}$ is a $p$-group. Suppose $\sigma \in \gamma_{n}$ and $q$ is a prime different from $p$ such that $\sigma^{q} \in S \gamma_{n+1}$. Then, for $g \in G$, $\langle[\sigma, g]\rangle=\langle[s, g]\rangle$ for some $s \in S$. This is because $[\sigma, g]^{q}=\left[\sigma^{q}, g\right]$ and $\gamma_{n+1}$ is $q$-divisible and central. It follows that if $F$ is a finitely generated subgroup of $G$ then there is a subgroup $R$ of $\gamma_{n}(F)$ which is generated by at most $r_{p}$ commutators of weight $n$ such that $\gamma_{n+1}(F) \leqslant[\langle R, S\rangle, F]$. We can now proceed as in the proof of the lemma in [2] to deduce that $\gamma_{n+1}(F)$ is generated by at most $\left(r_{p}+r\right)^{2} n$ commutators, as required.

Proof of the corollary. Note first that the finite rank case is an immediate consequence of the theorem, while an abelian group satisfies min if and only if it is periodic of finite rank with $r_{p}=0$ for almost all $p$. For the maximal condition, if $\gamma_{n}(G)=R \gamma_{n+1}(G)$ for some finitely generated subgroup $R$, then $\gamma_{n+1}(G)=[R, G]$ is also finitely generated, by the argument used in the above proof. If $\Gamma_{n}$ is minimax, we choose a finitely generated subgroup $S$ of $\gamma_{n}$ which generates a torsion-free subgroup of maximal rank modulo $\gamma_{n+1}$ and note that the integers $v_{k}(g)$ may be chosen to be $\pi$-numbers, where $\pi$ is a finite set of primes such that $\gamma_{n} / S \gamma_{n+1}$ is a $\pi$-group. Then, with the same notation, $[S, G] / T$ is a $\pi$-group of finite rank and so $[S, G]$ is minimax. Factoring, we may assume $S$ is central. It is clear that $\gamma_{n+1}$ is then a $\pi$-group of finite rank and so minimax. The case where $\Gamma_{n}$ is min-by-max is dealt with similarly, thus concluding the proof.

\section{REFERENCES}

1. D. J. S. Robinson, A property of the lower central series of a group, Math. Z. 107 (1968), $225-231$. 
2. H. Smith, Hypercentral groups with all subgroups subnormal II, Bull. London Math. Soc. 18 (1986) 343-348.

3. J. P. Williams, The join of several subnormal subgroups, Math. Proc. Cambridge Philos. Soc. 92 (1982), 391-399.

Mathematics Institute

University College

CARDIFF

WALES. 